\title{
Factores que determinan el uso de las TIC en adultos mayores de Chile
}

\author{
Factors that determine the use of ICTs in Chile's older adults
}

Factores determinantes para a utilização das TIC nos adultos mais vellhos do Chile

Sandra Marlene Aguilar-Flores ${ }^{1}$

Margarita María Chiang-Vega ${ }^{(1)}$

Recibido: marzo 2020

Aceptado: agosto 2020

Para citar este artículo: Aguilar-Flores, S. M., Chiang-Vega, M. M. (2020). Factores que determinan el uso de las TIC en adultos mayores de Chile. Revista Científica, 39(3), 296-308. https://doi.org/10.14483/23448350.16054

\section{Resumen}

El objetivo de este estudio es determinar los factores del uso de las tecnologías de la información y comunicación en los adultos mayores de la provincia de Concepción, Chile, basado en el modelo de la teoría unificada de adopción y uso de tecnología (UTAUT). El estudio se realizó sobre una población de 101 adultos mayores; para el análisis de los datos y comprobación de las hipótesis se ha estimado un modelo de ecuaciones estructurales. Las relaciones propuestas, utilizando variables latentes, contribuyen a explicar el uso de las tecnologías de la información en un $55 \%$ de los adultos mayores. Para disminuir la brecha digital se puede concluir que sí existen factores que determinan positivamente el uso de las TIC, lo que les facilita tener acceso de forma óptima. De este modo, mejoraría su calidad de vida y tendrían una real integración digital.

Palabras clave: adopción, adultos mayores Chile, UTAUT, tecnologías de la información.

\begin{abstract}
The objective of this study is to determine the factors of the use of information technologies in older adults in the province of Concepción, Chile, based on the model The Unified Theory of Adoption and Use of Technology (UTAUT). The study was carried out on the population of 101 older adults, for the analysis of the data and to check the hypotheses, a model of structural equations has been estimated. The proposed relationships, using latent variables, help to explain the Use of information technologies by $55 \%$ in older adults. To reduce the digital divide, it can be concluded that there are factors that positively determine the use of ICT, which makes it easier for them to have optimal access, thus improving their quality of life and real digital integration.
\end{abstract}

Keywords: adoption, seniors Chile, UTAUT, information technologies.

1. Universidad del BÍO BÍO, Chile. violetarifo@gmail.com

2. Universidad del BíO BíO, Chile.mchiang@ubiobio.cl 


\section{Resumo}

O objetivo deste estudo é determinar os fatores de uso das tecnologias da informação em idosos da província de Concepción, Chile, com base no modelo A teoria unificada da adoção e uso da tecnologia (UTAUT). O estudo foi realizado com a população de 101 idosos, para análise dos dados e verificação das hipóteses, um modelo de equações estruturais foi estimado. As relações propostas, utilizando variáveis latentes, ajudam a explicar o uso de tecnologias da informação em 55\% em idosos. Para reduzir o fosso digital, pode-se concluir que existem fatores que determinam positivamente o uso das TIC, o que facilita o acesso ideal, melhorando a qualidade de vida e a integração digital real.

Palavras-chaves: adoção, seniores Chile, UTAUT, tecnologias da Informação.

\section{Introducción}

El envejecimiento de la población es uno de los procesos globales de transformación social. Sus tendencias, iniciadas durante la fase de industrialización y posterior modernización de los países europeos, se han producido con diversos ritmos e intensidades por todo el planeta. Hace unas décadas la comunicación era realizada a través de cartas y llamadas a teléfonos fijos; en la sociedad actual comunicarse por medio de plataformas o redes sociales, de videollamadas, correo electrónico o el comercio electrónico B2C obedece al acelerado cambio de las tecnologías de la información y comunicación (TIC) que brindan oportunidades para poder estar conectados en cualquier parte del mundo con la familia y amigos. Para el adulto mayor esta brecha digital y la adopción de la tecnología es complejo en su diario vivir; pero el usar las herramientas que las TIC ofrecen mejorará su calidad de vida.

Niehaves y Plattfaut (2014) argumentan que el envejecimiento demográfico es una tendencia importante, en especial en las sociedades occidentales. Tiene implicaciones para las organizaciones públicas y privadas, especialmente para el grupo de personas mayores con diferentes actitudes, creencias e intenciones cuando se trata de uso de la tecnología (pp. 708-726). En general, se puede observar que los adultos mayores son más reticentes a utilizar las TIC; igualmente, la internet es significativamente menos usada por los ancianos que por otros grupos de edad. La forma en que en la década pasada se comunicaban las personas hoy es impensable, y todo ello gracias a las tecnologías que ofrece oportunidades de poder estar conectado por medio de redes sociales por motivos laborales o cualquier otro motivo.

Según Arnold et al. (2018), tanto para la sociedad como para las personas y sus entornos, el envejecimiento puede considerarse como una de las mayores cambios de la humanidad. Chile se encuentra en una fase avanzada de ese proceso, personas de 60 y más años cuentan —si se compara las generaciones precedentes - con niveles educacionales más elevados, mejores estados de salud y de satisfacción vital, intereses y posibilidades más diversificadas y que se encuentren paulatinamente más organizados y activos que sus ancestros.

En Chile estamos frente a una población que envejece de forma acelerada, según los datos entregados por el Instituto Nacional de Estadística de Chile (2019). Para el censo de 2017 el total de personas mayores de 60 años era de 2850 171, un 15,8 \% para el año 2020 llegaría a un 17,3 \% de la población (Amuch, 2017). Cada año son 100000 las personas que entran a formar parte del segmento adulto mayor. En las últimas décadas persiste la tendencia de que los adultos mayores cuentan con niveles educacionales más elevados; sin embargo, hay una gran parte de esta población que no tiene estudios, lo que implica que no tienen las competencias que les permitan usar las TIC.

Es incuestionable que las TIC ofrecen oportunidades y beneficios, ¿estas oportunidades benefician a este grupo etario de la sociedad chilena? Para dar respuesta a esta pregunta, el objetivo del estudio es conocer los factores que determinan el uso de las tecnologías de información en los adultos mayores 60 años mujeres y 65 años hombres en Chile, mediante el modelo UTAUT. 
Se espera que el aumento del uso de las TIC impacte positivamente al bienestar de los adultos mayores. Involucrarlos en la era digital trae muchos beneficios tanto para su inserción social como para su salud física y mental, lo que ofrecería mayores posibilidades de inclusión laboral y una real integración al desarrollo de nuestra sociedad digital. Acortar esa brecha tanto generacional como digital es tarea de todos.

\section{Redes sociales}

Sanz-Menéndez (2003) define que las redes sociales como un conjunto limitado de actores vinculados unos a otros a través de una relación. Las redes sociales constituyen una estructura que organiza la relación entre las personas y la forma en que la información circula, es así que toda red implica una trama social y de comunicación. Ellas han marcado el ritmo de nuestra sociedad, desarrollando un cambio en la dinámica social.

Para Díaz-Llairó, (2011) "las redes sociales son espacios en la red de internet que cuentan con herramientas tecnológicas sencillas de utilizar que permiten el intercambio entre las personas para conocerse, ofrecer servicios realizar negocios; compartir e intercambiar información"(pp. 44-45). Hoy en día, la tecnología presenta múltiples herramientas que transforma el modo en que los actores se comunican y relacionan.

\section{Teoría unificada de la aceptación del uso de la tecnología (UTAUT)}

Arenas-gaitán et al., (2016) explican que el modelo de la teoría unificada de la aceptación del uso de la tecnología (UTAUT, por sus siglas en inglés) se compone de modelos y teorías que se plantearon para analizar la aceptación del usuario de una tecnología. Este modelo integrador propuesto por Venkatech et al. (2003) combinó los ocho modelos que competían por explicar la adopción de TIC en usuarios. A saber: teoría de difusión de innovaciones (IDT); teoría social cognitiva (SCT); teoría de acción razonada (TRA); modelo de utilización del PC (MPCU); modelo de aceptación tecnológica (TAM); modelo motivacional (MM); teoría del comportamiento planeado (TPB) y una combinación de TAM y TPB (C-TAM-TPB). Además, se identificaron cuatro variables moderadoras significativas: género, experiencia, edad y voluntariedad de uso (Taherdoost, 2018).

Para Venkatesh (2008), la investigación sobre la adopción de TIC en el ámbito individual ha sido bastante estudiada y ha proporcionado teorías y explicaciones de los determinantes de la adopción y la decisión de uso de la tecnología. Cataldo y Muñoz (2013) explican que este modelo es uno de los que mejor interpreta la adopción de un sistema, llegando a niveles del $70 \%$ de predictibilidad.

El modelo UTAUT propone que las expectativas de desempeño, las expectativas de esfuerzo y la influencia social afectan a la intención de uso; mientras que las condiciones facilitadoras determinan el uso efectivo de la nueva tecnología (Almenara et al., 2016). A continuación, se detallan las características de la UTAUT:

1. Las expectativas de desempeño (Performance Expectancy, PE): hace referencia al nivel en que un individuo percibe que el uso de la tecnología le será beneficioso en el desempeño laboral o profesional. Es similar al factor de utilidad percibida del modelo TAM, pues tiene que ver con la motivación extrínseca, la ventaja relativa de la tecnología y las expectativas de desenlace. Se considera el factor más importante e influyente con respecto a la intención de comportamiento, tanto en escenarios donde el uso sea voluntario como obligatorio.

2. Las expectativas de esfuerzo (Effort Expectancy, EE): esto implica el nivel de facilidad de uso asociado al uso de la tecnología. Tiene que ver con el factor de facilidad de uso percibida del modelo TAM y lo complejo de la tecnología. Este constructo es también importante en contextos donde el uso sea obligatorio o 
voluntario, pero solamente en relación con la experiencia de uso en términos de cantidad. Este factor es más influyente en mujeres que en hombres, así como en los adultos mayores; es decir, estos usuarios son más propensos a ser influenciados por el esfuerzo percibido que implica el uso de la tecnología.

3. La influencia social (Social Influence, SI): se refiere al nivel en que la persona percibe que los demás valorarán la utilización de la tecnología, tanto de personas consideradas influyentes como importantes; el individuo percibe que el comportamiento de otros como los amigos la familia, es valioso.

4. Las condiciones facilitadoras (Facilitating Conditions, FC): tiene que ver con el nivel en que una persona considera que existe la estructura organizativa y técnica que soporte la adopción de la tecnología. Tienen que ver con el control conductual percibido con base en la existencia de condiciones como conocimiento y compatibilidad, formación por parte de la organización, soporte técnico y otros recursos. Se considera que si estas condiciones son satisfechas se remueven las barreras de uso. Este constructo influye directamente sobre el comportamiento de uso y tiene un efecto mayor en los adultos mayores y en personas con más experiencia de uso.

Los primeros tres constructos son determinantes directos de las intenciones y comportamiento de uso, mientras que el cuarto es determinante del comportamiento de uso. En este modelo, el género (sexo), la edad, la experiencia y la voluntad de uso se plantean como moderadores del impacto de los cuatro constructos en la intención de uso y comportamiento. Tanto Venkatesh et al. (2003) como Chen y Chan (2014) explican que el valor del modelo UTAUT reside en que identifica los principales determinantes de la adopción, así como permite incluir y considerar el efecto de diferentes moderadores que afectan en la influencia de los constructos claves del modelo.

\section{Adulto mayor y las TIC}

La Organización Mundial de la Salud (2015) define el envejecimiento como el proceso fisiológico del ser humano desde la concepción hasta la muerte con cambios durante todo el ciclo de la vida (como se cita en Mar et al., 2014). El envejecimiento es un proceso complejo de la vida, donde se experimentan cambios de orden físico, psicológico y social. En Chile, el aumento de la esperanza de vida en las últimas décadas ha aumentado. Arnold et al. (2018) y Selwyn (2004) explican que para los adultos mayores la exclusión social se da por desigualdades en la edad y el nivel educacional, lo que se traduce en bajos ingresos para cualquier género de este grupo etario.

Por su parte, en (Selwyn, 2004)un extenso estudio que trata de desarrollar una teoría unificada de aceptación y uso de la tecnología, Venkatesh et al. (2003) investigó el impacto moderador de la edad en las relaciones teóricas clave de la aceptación de la tecnología, encontrando que el efecto de la expectativa de desempeño en la intención de comportamiento que se usa es más fuerte para los trabajadores más jóvenes. Mientras que el efecto encontrado en la expectativa de esfuerzo y la influencia social sobre la intención de uso del comportamiento y el de facilitar las condiciones sobre el uso real fueron más fuertes para los trabajadores de mayor edad. De modo que es evidente que la edad sí afecta las actitudes y comportamientos de los usuarios relacionados con la aceptación y el uso de la tecnología.

Hong et al. (2013) describen que las diferencias de edad desempeñan una tarea importante en la comprensión de las percepciones y conductas humanas en diversos dominios de investigación, incluida la psicología, la conducta organizativa y la comercialización. El papel de la edad es igual o más importante para la investigación de sistemas de información, ya que el comportamiento real, así como la actitud con respecto a la adopción de tecnología están vinculados de manera crítica con la edad del usuario. Además, debido al rápido 
desarrollo y la introducción de nuevas tecnologías, diferentes generaciones de edades se enfrentan inevitablemente a diferentes generaciones de tecnologías. Por ejemplo, la mayoría de los adolescentes de hoy crecieron con la interfaz gráfica de usuario (GUI), así como una predominancia de lo visual y nunca han usado interfaces de línea de comandos (CLI). El correo electrónico es el medio de comunicación preferido por los usuarios de hoy en día, mientras que aquellos que ingresan a la fuerza laboral prefieren interactuar entre sí a través de redes sociales interactivas como Facebook, Instagram o Twitter; las actitudes hacia la tecnología pueden variar dependiendo de la edad.

Sin embargo, a pesar de su importancia en la adopción y el uso de la tecnología, la edad ha recibido poca atención en la investigación. La edad puede ser problemática a medida que la población envejece y las TIC se vuelven más omnipresentes y forman parte integral de muchos productos y servicios cotidianos, con la separación entre el trabajo y el uso personal de dichos sistemas.

Venkatesh et al. (2014) estudiaron la influencia de la edad en la adopción de tecnologías en un entorno organizativo. Encontraron que la facilidad de uso percibida y la norma subjetiva tienen una influencia más destacada en la decisión de adopción de tecnología inicial para los trabajadores mayores que para los trabajadores más jóvenes. Sin embargo, se encontró que este efecto de contraste disminuye con el tiempo a medida que los trabajadores ganan más experiencia en el uso del sistema. Morris et al. (2003) encontraron que la edad tiene un impacto moderador significativo en las relaciones claves en la teoría del comportamiento planificado con la edad, se puso un mayor énfasis en la actitud hacia el uso de la tecnología en las decisiones relativas a la adopción de tecnología. Este efecto varió según el género, con un efecto más fuerte para los hombres en comparación con las mujeres. En contraste, se encontró que el impacto de la norma subjetiva en la aceptación de la tecnología era más fuerte con el aumento de la edad. Sin embargo, este efecto solo se encontró para la población usuaria femenina, pero no para los hombres.

Cresci et al. (2010), Ramón-Jerónimo (2013) y McCloskey (2006) estudiaron a un grupo significativo de adultos mayores conectados con internet y que usan sus herramientas y aplicaciones. El mayor porcentaje es ocupado por aquellos que tienen una menor edad dentro de los adultos mayores, esto explica cómo la edad cronológica en este grupo lleva a diferencias en su comportamiento en internet. Así, la probabilidad de realizar una transacción en línea es menor para aquellos adultos de la cuarta edad que además perciben mayor dificultad en la utilización de internet. En general, el uso de internet se reduce a medida que aumenta la edad, en especial los adultos que tienen o superan los 70 años (Eurostat, 2005).

Condeza et al. (2016) sugieren el aprendizaje más activo de las TIC en los adultos mayores. Por otro lado, Llorente-Barroso et al. (2015) indican que las oportunidades que ofrece internet a los adultos mayores se centran en cuatro categorías: informativas, comunicativas, transaccionales y de entretenimiento. Estudios han demostrado que la participación de los adultos mayores en programas de alfabetización digital incrementa el uso de las TIC como un instrumento para mejorar su calidad de vida facilitando la respuesta a sus necesidades y el acceso a la información disponible en la red (Casado-Muñoz et al., 2015).

\section{Metodología}

Para cumplir con el objetivo de la investigación se realizó un estudio de diseño no experimental, de tipo transversal y causal. La muestra está formada por 101 adultos mayores de 60 años si es mujer y 65 años si es hombre, de la provincia de Concepción, Chile. El modelo teórico aplicado con base en la literatura revisada de la UTAUT son: expectativas de desempeño (PU), expectativas de esfuerzo (PEOU), condiciones facilitadoras e influencia social; se utilizó la escala de Venkatesh et 
al. (2003). Se postularon las siguientes hipótesis en la investigación:

- H1: a mayor percepción de utilidad de la TIC, mayor intención de uso de las TIC por parte de los adultos mayores.

- H2: a mayor percepción de facilidad de uso de las TIC, mayor intención de uso de las TIC por parte de los adultos.

- H3: a mayores condiciones facilitadoras, mayor intención de uso de las TIC en los adultos mayores.

- H4: a mayor intención de uso de las TIC, mayor uso de las TIC por parte de los adultos mayores.

- H5: a mayor influencia social de uso de las TIC por parte de los adultos mayores, mayor intención de su uso.

\section{Resultados}

La base de datos está conformada por $n=101$ adultos mayores, los cuales fueron seleccionados por conveniencia (los adultos mayores que aceptaron participar de forma voluntaria) para contestar la encuesta. Se midieron tres indicadores sociodemográficos y se aplicó escala referente a actitudes respecto del uso de TIC. Para el tratamiento de los datos se realizó el análisis de fiabilidad mediante el alfa de Cronbach (medidos en escala tipo Likert). La descripción de las variables se muestra en la tabla 1.

El promedio de edad de las mujeres es de $71,27 \pm 6,3$ años, y de los hombres es de 74,75 \pm 7,6 años. Esta diferencia es estadísticamente significativa al $5 \%\left(t=-2,75 ; \mathrm{p}=0,007^{* *}\right)$. Respecto a la cantidad de años utilizando TIC, las mujeres promediaron 8,03 \pm 6,4 años; mientras que los hombres 11,91 $\pm 9,9$ años. Esta diferencia también es significativa al $5 \%(t=-3,36 ; p=0,002 * *)$. Esto implica que los hombres tienen una mayor experiencia en el uso de TIC, cosa que se explica dado al estilo de vida que se daba en aquellas épocas. También se destaca que la cantidad de años que las utilizan es bastante pequeño comparado con su edad; es decir, solo alrededor de los 60 años de edad comenzaron a utilizar la tecnología.

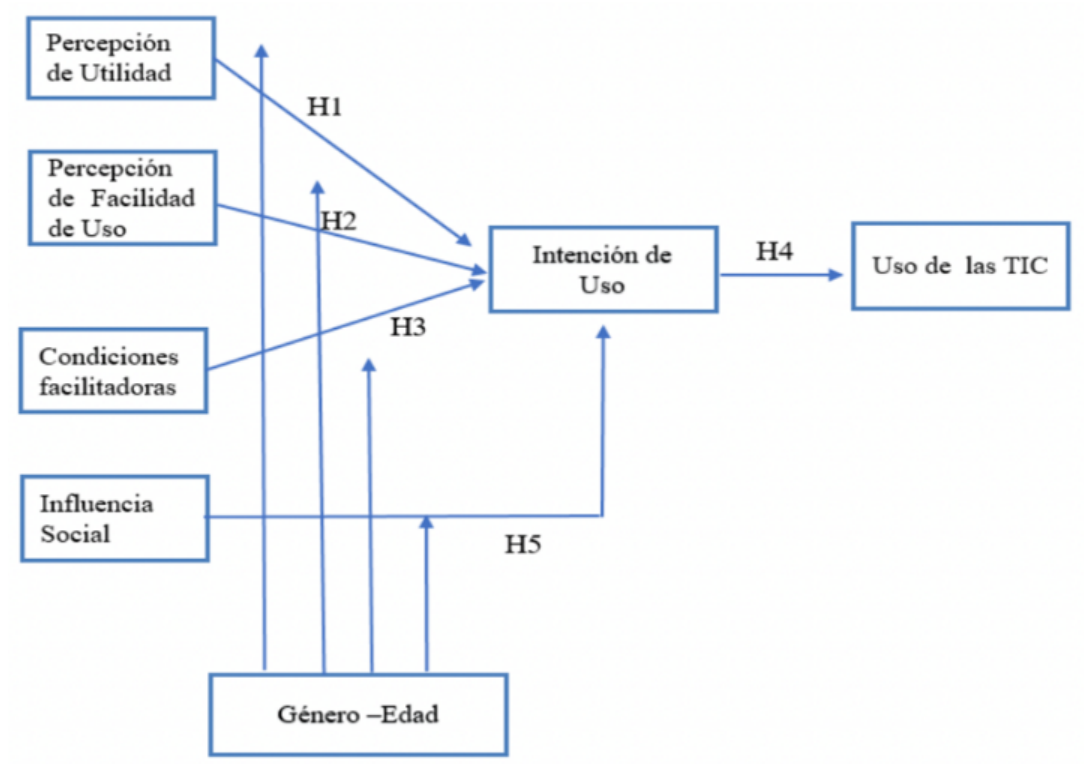

Figura 1. Modelo teórico UTAUT propuesto sobre los factores que influyen en el uso de las TIC en los adultos mayores de la provincia de Concepción, Chile.

Fuente: elaboración propia. 
Tabla 1. Mediciones realizadas

\begin{tabular}{llll}
\hline Variable & Tipo & Características & Número de ítems \\
\hline Edad & Sociodemográfica & $\begin{array}{l}\text { Prom=73,0 } \pm 6,9 ; \\
\text { Mínimo=61; Máximo=90 }\end{array}$ & - \\
\hline Género & Sociodemográfica & $\begin{array}{l}\mathrm{n}=70 \text { femenino; } \\
\mathrm{n}=31 \text { masculino }\end{array}$ & - \\
\hline Nivel educacional & Sociodemográfica & $37 \%$ enseñanza media & - \\
\hline Uso de TIC & Variable dependiente & Prom=4,2 $\pm 1,5$ & - \\
\hline Percepción de utilidad & Variable independiente & Prom=15,5 $\pm 2,7$ & 3 ítems (P7, P8, P9) \\
\hline $\begin{array}{l}\text { Percepción de facilidad de } \\
\text { uso }\end{array}$ & Variable independiente & Prom=10,3 $\pm 4,1$ & 3 ítems (P10, P11, P12) \\
\hline Condiciones facilitadoras & Variable independiente & Prom=13,1 $\pm 3,5$ & 3 ítems (P20, P21, P22) \\
\hline Influencia social & Variable independiente & Prom=16,4 $\pm 2,3$ & 3 ítems (P23, P24, P25) \\
\hline Intención de uso & Moderadora & Prom=13,6 $\pm 3,9$ & 3 ítems (P14, P15, P16) \\
\hline
\end{tabular}

Fuente: elaboración propia.

\section{Modelos de ecuaciones estructurales}

Se utilizaron ecuaciones estructurales dado que el modelo evidenció las hipótesis propuestas. No se aplicó validez convergente (si todos los ítems cargan a una sola columna del análisis factorial exploratorio) y discriminante (la carga de las otras subescalas sea despreciable) para utilizar ecuaciones estructurales, ya que la validez convergente y discriminante se utiliza cuando la escala tiene subescalas. En este caso, son todas escalas unidimensionales (que no se dividen en subescalas), las cuales se escogieron para proponer relaciones de causa efecto de acuerdo con el marco teórico. Además, este proceso se realiza en la etapa de validación, que es dentro de la creación de las escalas y estas ya fueron objeto de validación en trabajos anteriores (Peral et al., 2014; Grandon et al., 2019).

Para comprobar las hipótesis se ha estimado un modelo de ecuaciones estructurales utilizando variables latentes. El modelo conceptual previo a la estimación es el presentado en la figura 2, al momento de estimar tanto el modelo de medida como el modelo estructural, los p-valor se han obtenido recursivamente mediante bootstrapping con $M=5000$ muestras. Para la bondad de ajuste global se han considerado los índices RMSEA y el porcentaje de errores estandarizados absolutos inferiores a los cálculos han sido realizados mediante el software AMOS 22.0.

El bootstrapping (o remuestreo) es un método que se utiliza para combatir la ausencia de normalidad, fenómeno que es recurrente en la práctica e invalida ciertas metodologías. Consiste en realizar sucesivas submuestras dentro de las observaciones de la muestra obtenida, a fin de conseguir los coeficientes del modelo en cada una de las submuestras estas submuestras generalmente se generan de forma masiva y la cantidad se elige de manera arbitraria (100, 1000, 5000, etc.) pudiéndose obtener intervalos de confianza no-paramétricos al $95 \%$, además de los p-valor para evaluar significancia estadística. En este caso se han seleccionado 5000 submuestras.

Los elementos de los modelos de ecuaciones estructurales se pueden explicar de la siguiente forma:

- Modelo de medida: relaciona la variable latente con sus ítems, el objetivo es determinar si la variable se manifiesta de forma significativa sobre sus ítems, es decir, que cada uno es un buen indicador de la variable definida. Es 


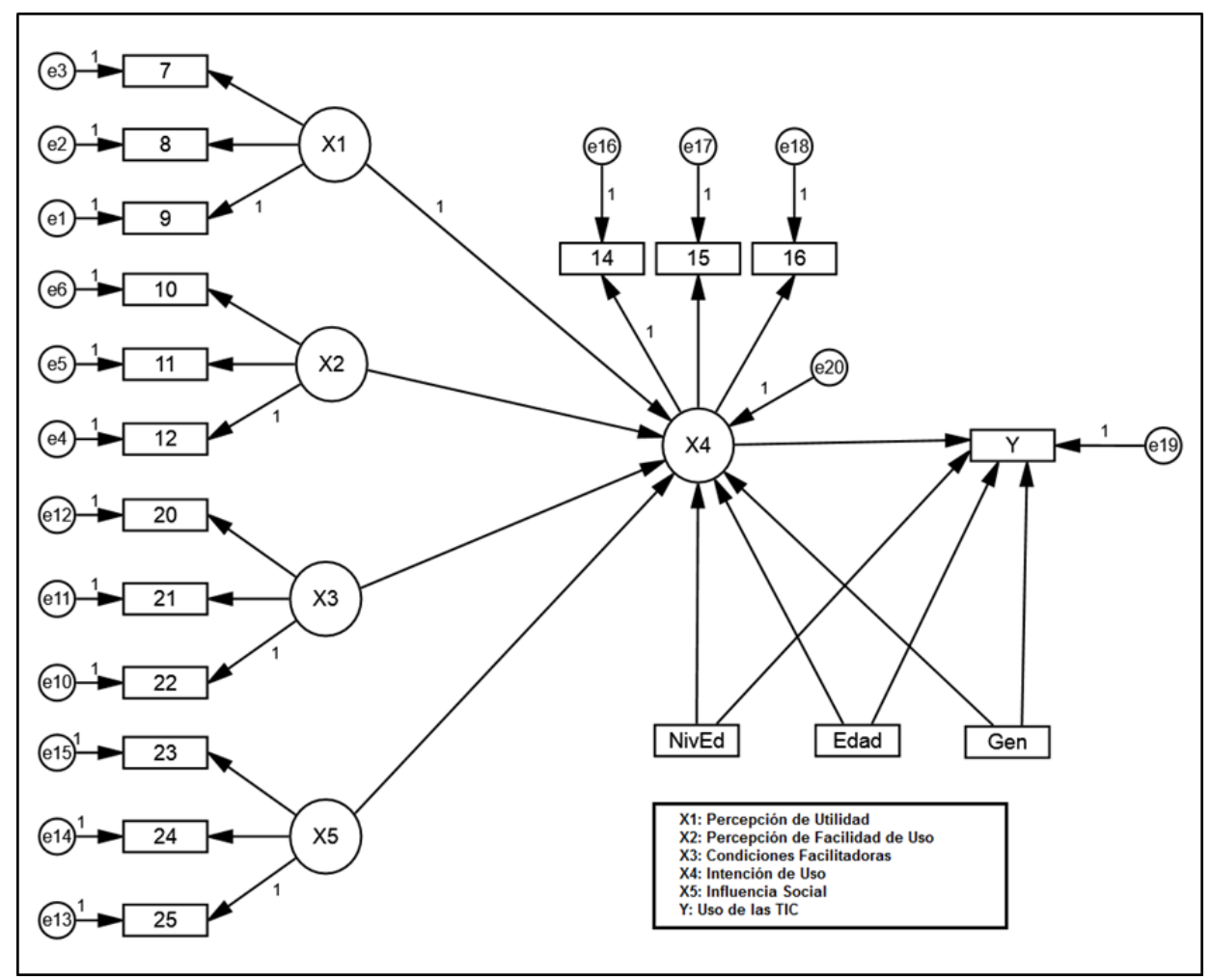

Figura 2. Modelo conceptual.

Fuente: elaboración propia.

aquella parte del modelo general en la cual solo se considera la relación entre las variables y sus indicadores.

- Modelo estructural: permite comprobar hipótesis respecto a las variables latentes definidas, responden a relaciones causa-efecto y que pueden ser uni o bidireccionales. Solo considera las relaciones entre variables latentes y covariables (edad, género, nivel educacional).

En la tabla 2 se muestran los resultados del modelo de medida, donde se observa que los coeficientes de los ítems pertenecientes a cada variable se comportan de manera adecuada, ya que son en su mayoría superiores a 0,6 y estadísticamente significativos al $5 \%$, lo que indica que las variables se manifiestan de manera correcta en los ítems que pretenden medirlas. En cuanto las propiedades globales, se tiene que los índices de fiabilidad compuesta (IFC) son superiores a 0,7 (excepto para influencia social), mostrando que este último no es un indicador tan confiable para medir tal variable. Con respecto a la validez, se tiene que las escalas son válidas a excepción de percepción de utilidad e influencia social; esto implica que el grado de pertinencia de los indicadores no es suficiente para que sea representativo.

\section{Comprobación de hipótesis}

A continuación, se observa que para las hipótesis $\mathrm{H} 1, \mathrm{H} 2, \mathrm{H} 3, \mathrm{H} 4$ y $\mathrm{H} 5$ son todos coeficientes positivos y con fuerte evidencia $(p<0,1)$. Se da cuenta de que las variables que cumplen con sus hipótesis respectivas hacen incrementar la intención de uso de las TIC; lo que apoya al modelo propuesto y revela que la intención de uso, en definitiva, hace que aumente el uso de las TIC. Con respecto a las variables sociodemográficas, ninguna de ellas tiene impacto sobre la intención de uso ni el uso de las TIC. 
Tabla 2. Resultados modelos de medidas

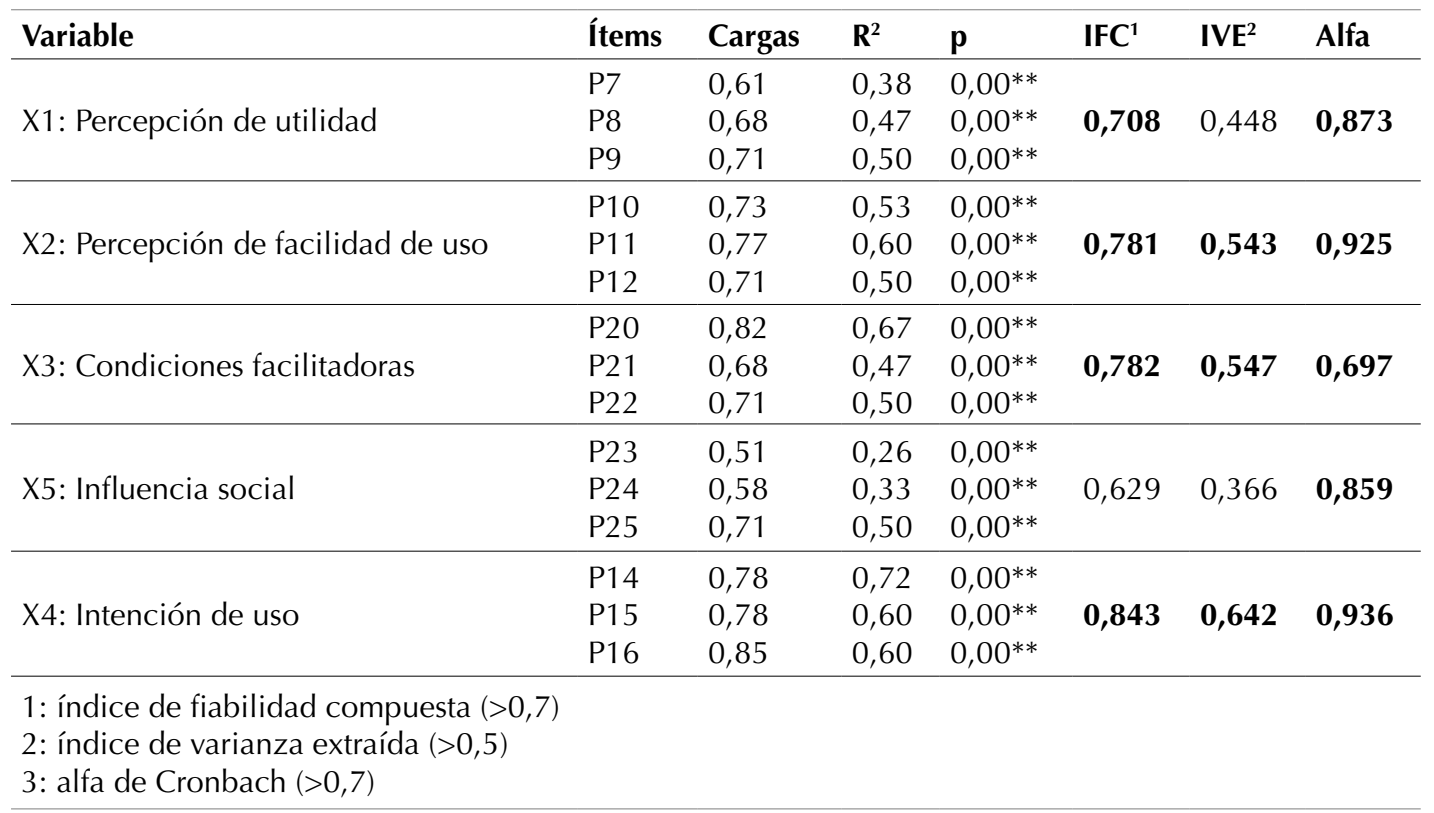

Fuente: elaboración propia.

Tabla 3. Estimaciones obtenidas

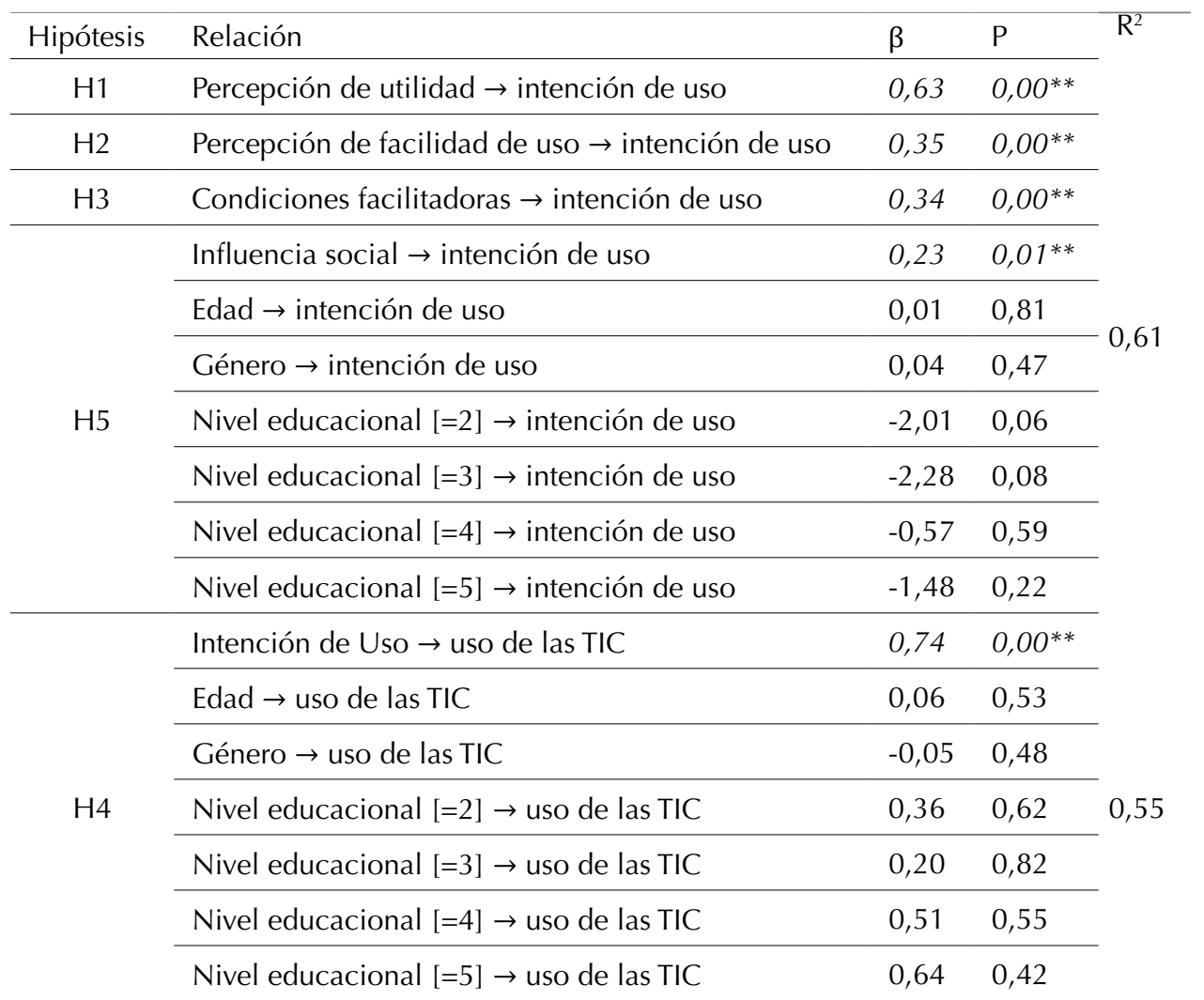

Fuente: elaboración propia. 
Respecto a la bondad de ajuste, de la tabla 3 se tiene que el porcentaje de contribución (R2) de las variables explicativas que afectan a la variable mediadora intención de uso es de un $61 \%$; esto significa que las variables en conjunto aportan un $61 \%$ a explicar los cambios en la intención de uso. Mientras que para la variable uso de TIC es de un $55 \%$. Con respecto a las estimaciones internas, se tiene un RMSEA (indicador de cuanto se equivoca el modelo en general) de 0,12, el cual es levemente superior al umbral máximo de 0,10 ; lo que indica que hay un ajuste aceptable pero no óptimo. El porcentaje de residuos absolutos estandarizados menores a 2 es de un 84,2 \%, esto revela que el modelo es capaz de replicar bien la variabilidad maestral y de replicar la información muestra inicial; con estos datos es posible predecir el comportamiento futuro en igual contexto.

\section{Discusión}

Para la comprobación de las hipótesis planteadas en esta investigación se encontró que las variables que cumplen el caso de percepción de utilidad son las tres cargas superiores a 0,6 y son estadísticamente significativas al $1 \%$; lo que señala que los ítems individualmente representan bien al constructo. Con respecto al $\mathrm{R}^{2}$ la percepción de utilidad, este contribuye entre un $38 \%$ y un $50 \%$ a explicar que los ítems que la representan hacen incrementar la intención de uso de las TIC en un $61 \%$, apoyando el modelo propuesto y revelando que la intención de uso en definitiva hace que aumente el uso de las TIC. Condeza et al. (2016) sugieren que para la incorporación más activa de las TIC (y el querer integrarse a la tecnología más reciente) se debería procurar, en la medida de lo

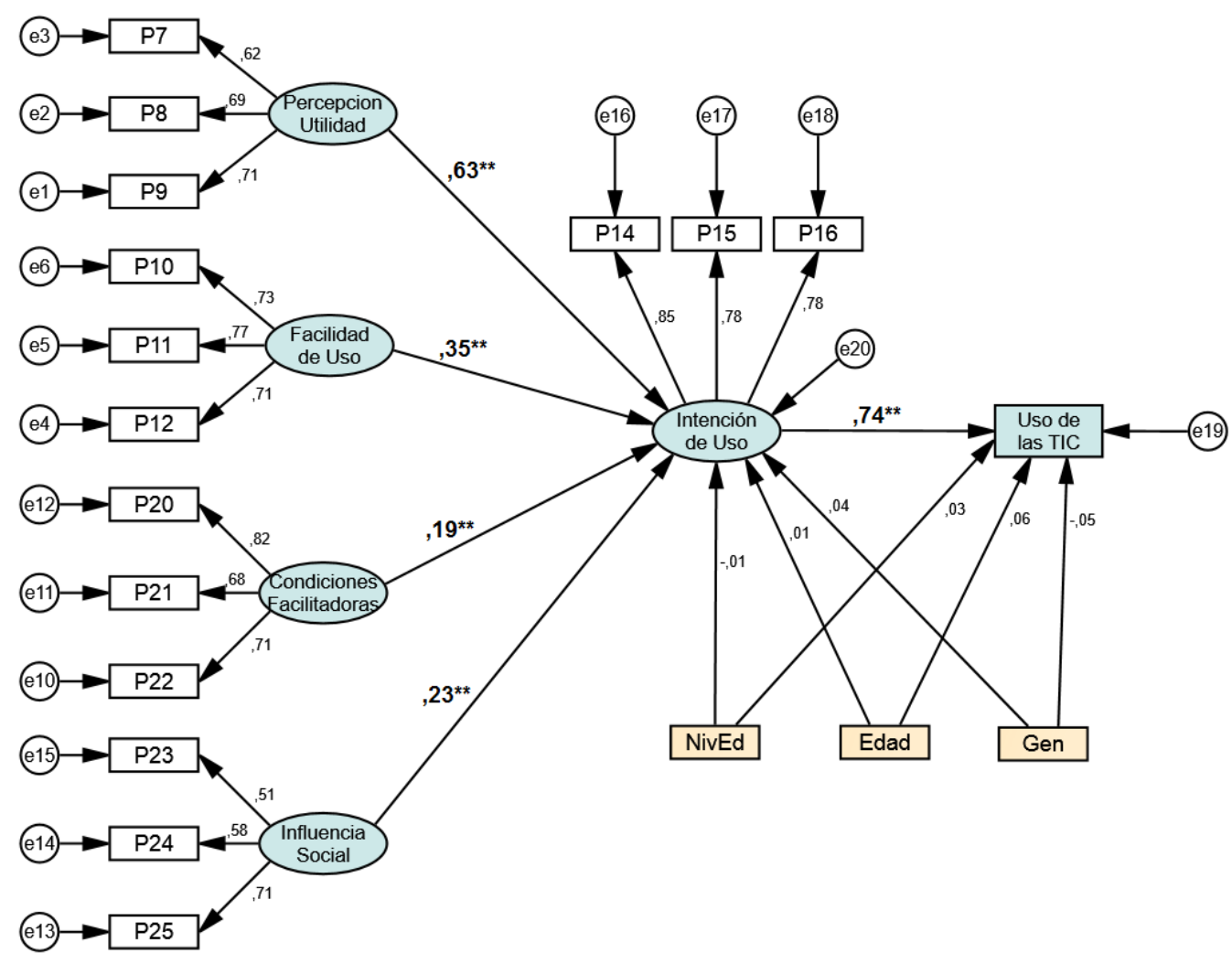

Figura 3. Modelo estimado donde se comprueban todas las hipótesis.

Fuente: elaboración propia. 
posible, que las personas se sienta más útiles y que pueden aportar.

Tal como indican Grandon et al. (2019), los adultos mayores que más utilizan las redes sociales son los que confían en sí mismos, lo que les permite percibir que es fácil de usar; en esa investigación se obtuvo como resultados en PU y PEOU el $38 \%$ del uso de redes sociales por parte del adulto mayor, lo que respalda el modelo TAM. Comparando estos resultados con los obtenidos en la presente investigación se observa que las dos variables explicativas intención de uso y uso contribuyen a explicar significativamente el uso de las TIC con un aporte $\left(\mathrm{R}^{2}\right)$ del 55 \%. Abad (2014) precisa que para una mejor calidad de vida en la tercera edad son necesarios programas de alfabetización digital que se centren en la formación de competencias para los adultos mayores, lo que se conoce como e-inclusión.

\section{Conclusiones}

El envejecimiento en Chile es una realidad: si bien el país aún no se encuentra en una etapa avanzada del envejecimiento poblacional en comparación con otros países desarrollados, se prevee que la población mayor de 65 años crezca rápidamente en los próximos 20 años, por lo que se espera que los adultos mayores pasen de ser un $10 \%$ del total de la población en el 2010 a un $20 \%$ en el 2038 (Villalobos-Dintrans, 2017; Madariaga, 2001). ¿Cómo se va a enfrentar esta situación si ya ahora los adultos mayores requieren ayuda para comunicarse, comprar o buscar información? Una realidad que podría ayudar a solucionar el problema es la alfabetización digital de los adultos mayores, pues ellos pueden continuar aprendiendo, adquirir conocimientos y capacidades para controlar su salud, mantenerse al día respecto de los avances en materia de información y tecnología, participar (por ejemplo, mediante el trabajo o el voluntariado), adaptarse al envejecimiento (por ejemplo, a la jubilación, a la viudez o a cuidar de otra persona), mantener su identidad y conservar el interés en la vida. Las capacidades para aprender, crecer y tomar decisiones están muy vinculadas a la autonomía, la dignidad, la integridad, la libertad y la independencia de los adultos mayores (Boulton-Lewis, 2010; Welford et al., 2010; Lindberg et al., 2014).

Desde estas ideas surge la necesidad de conocer acerca de las posibilidades que brindan las TIC para disminuir la brecha digital en los adultos mayores de la provincia de Concepción en Chile. Al considerar que impactan positivamente a su bienestar social, económico y de ocio, dado el aumento de la población en este grupo etario en Chile (Amuch, 2017), es importante la inclusión del adulto mayor y las TIC ya que se mejora su calidad de vida y con ello se alargan sus años de independencia.

Esta investigación buscó analizar los factores del uso de las tecnologías de la información en los adultos mayores, por medio del modelo UTAUT. Las hipótesis se comprobaron a través de modelos de ecuaciones estructurales de las relaciones propuestas entre variables actitudinales respecto al uso de las tecnologías de la información en adultos mayores, revelando que las cuatro variables explicativas contribuyen a entender significativamente la intención de uso con un aporte $\left(\mathrm{R}^{2}\right)$ del $61 \%$ y la intención de uso explica un $55 \%$ del uso de las TIC. Las variables de control nivel educacional, la edad y el género no son factores que promuevan la intención de uso ni el uso de las TIC, convirtiéndolos en fenómenos transversales a este grupo etario.

Para que cualquier persona independiente de su edad pueda utilizar las diversas tecnologías que han facilitado las actividades del día a día como comunicarse por mensajes, transacciones bancarias, compra de artículos, etc., deben estar diseñadas para que el adulto mayor pueda utilizarlas. Es por esto por lo que, si los adultos mayores consideran que las tecnologías son fáciles de usar, se motivarían para aprender a utilizar las TIC (Fernández-Morales et al., 2016).

Se puede así concluir en este estudio que sí existen factores que determinan positivamente el uso 
de las TIC; factores que influyen en las intenciones y la relación de intención-comportamiento con lo cual se puede disminuir la brecha digital en los adultos mayores. Estos factores, así como la percepción de utilidad y la percepción de facilidad de uso, son posibles de intervenir por medio de la e-inclusión a través de la alfabetización digital; de modo que luego estas personas podrán tener acceso en forma óptima a las TIC, lo que mejoraría su situación personal y social. La limitación en esta investigación fue el tamaño de la muestra, dado que la información recolectada fue de una provincia y en el futuro se podría aumentar el alcance de este estudio, replicarlo y extender el estudio a más zonas del país; con ello, los resultados logrados serían más representativos para sugerir nuevas políticas de alfabetización digital para los adultos mayores.

\section{Referencias}

Abad, L. (2014). Diseño de programas de e-inclusión para alfabetización mediática de personas mayores. Media Literacy for Older People Facing the Digital Divide: The e-Inclusion Programmes Design, 21(42), 173-180. https://doi.org/10.3916/C42-2014-17

Almenara, J. C., Osuna, J. B., Cejudo, M. del C. L. (2016). Technology acceptance model \& realidad aumentada: Estudio en desarrollo. Revista Lasallista de Investigacion, 13(2), 18-26. https://doi.org/10.22507/rli.v13n2a2

Amuch (2017). Los adultos mayores en las comunas de Chile : actualidad y proyecciones. http:// www.amuch.cl/wp-content/uploads/2017/05/ Estudio Adulto Mayor en las comunas de Chile Proyecciones

Arnold, M., Herrera, F., Massad, C., Thumala, D. (2018). Quinta encuesta nacional y exclusión social de las personas mayores en Chile 2017. Ediciones Servicio Nacional del Adulto Mayor. Boulton-Lewis, G. M. (2010). Education and Learning for the Elderly: Why, How, What, Educational Gerontology, 36(3), 213-228. https://doi.org/10.1080/03601270903182877
Cataldo, C. A., Muñoz, C. N. (2013). Validación cualitativa de UTAUT Evidencias desde un estudio de investigación acción. Infonor. IV Congreso Internacional de Computación e Informática Del Norte de Chile (pp. 1-9). https://doi.org/10.13140/2.1.4446.9767

Chen, K., Chan, A. H. S. (2014). Predictors of gerontechnology acceptance by older Hong Kong Chinese. Technovation, 34, 126-135. https:// doi.org/10.1016/j.technovation.2013.09.010

Condeza, A. R., Bastías, G., Valdivia, O., Cheix, C., Barrios, X., Rojas, R., Gálvez, M., Fernández, F. (2016). Adultos mayores en Chile: descripción de sus necesidades en comunicación en salud preventiva. Cuadernos.Info, 38, 85-104. https://doi.org/10.7764/cdi.38.964

Cresci, M. K., Yarandi, H. N., Morrell, R. W. (2010). Pro-nets versus no-nets: Differences in urban older adults' predilections for internet use. Educational Gerontology, 36(6), 500-520. https://doi.org/10.1080/03601270903212476

Díaz-Llairó, A. (2011). El talento está en la red. LID Editor.

Eurostat (2005). The digital divide in Europe. Animal Behaviour, 74(3), 359-361. https://doi.org/10.1016/j.anbehav.2007.06.003

Fernández-Morales, K., McAnally-Salas, L., Vallejo-Casarín, A. (2016). Apropiación tecnológica:Una visión desde los modelos y las teorías que la explican. Perspectiva Educacional, 54(2). https://doi.org/10.4151/07189729-vol.54iss.2-art.331

Grandon, E. E., Ordenes, L. B., Araya, S., Ramirez-Correa, P., Alfaro-Perez, J. (2019). Social network sites and elders: An empirical study. 2019 14th Iberian Conference on Information Systems and Technologies (CISTI), (pp. 1-4). https://doi.org/10.23919/CISTI.2019.8760617

Hong, S. J., Lui, C. S. M., Hahn, J., Moon, J. Y., Kim, T. G. (2013). How old are you reaIly? Cognitive age in technology acceptance. Decision Support Systems, 56(1), 122-130. https://doi.org/10.1016/j.dss.2013.05.008 
Instituto Nacional de Estadística de Chile (2019). https://www.ine.cl/estadisticas/laborales/ene

Lindberg, C., Fagerström, C., Sivberg, B. W. (2014). Concept analysis: patient autonomy in a caring context. Journal of $\mathrm{Ad}$ vanced Nursing, 70(10), 2208-2221. https://doi.org/10.1111/jan.12412

Llorente-Barroso, C., Viñarás-Abad M., Sánchez-Valle, M. (2015). Mayores e internet: la red como fuente de oportunidades para un envejecimiento activo. Comunicar, 23(45), 29-36. https://doi.org/10.3916/C45-2015-03

Madariaga, A. V. (2001). Envejecimiento $y$ vejez en América Latina y el Caribe: políticas públicas y las acciones de la sociedad. En Población y desarrollo. https://www.gerontologia.org/portal/archivosUpload/viveros.pdf

Mar, A., Garc, A., Maya, S. (2014). Análisis del concepto envejecimiento. Gerokomos, 25(2), 57-62. https://doi.org/10.4321/ $\underline{\mathrm{S} 1134-928 \times 2014000200002}$

McCloskey, D.W. (2006). The Importance of Ease of Use, Usefulness, and Trust to Online Consumers. Journal of Organizational and End User Computing, 18(3), 47-65. https://doi.org/10.4018/joeuc.2006070103

Niehaves, B., Plattfaut, R. (2014). Internet adoption by the elderly: Employing IS technology acceptance theories for understanding the age-related digital divide. European Journal of Information Systems, 23(6), 708-726. https://doi.org/10.1057/ejis.2013.19

Organización Mundial de la Salud (2015). Informe Mundial sobre el envejecimiento y la salud. https://doi.org/ISBN 978924356504

Peral, B. P., Gaitán, J. A., Ramón-Jerónimo, M. Á. (2014). Technology Acceptance Model y mayores: ¿la educación y la actividad laboral desarrollada son variables moderadoras? Revista Española de Investigación de Marketing ESIC, 18(1), 43-56. https://doi.org/10.1016/ s1138-1442(14)60005-x

Ramón-Jerónimo, M. A., Peral-Peral, B., Arenas-Gaitán,J.(2013).ElderlyPersonsand InternetUse.Social Science Computer Review, 31(4), 389-403. https://doi.org/10.1177/0894439312473421

Sanz-Menéndez, L. (2003). Análisis de Redes sociales: o como representar las estructuras sociales subyacentes. Apuntes de Ciencia y Tecnologia, 7, 21-27.

Selwyn, N. (2004). Reconsidering political and popular understandings of the digital divide. New Media and Society, 6(3), 341-362. https://doi.org/10.1177/1461444804042519

Taherdoost, H. (2018). A review of technology acceptance and adoption models and theories. Procedia Manufacturing, 22, 960-967. https://doi.org/10.1016/j.promfg.2018.03.137

Venkatesh,M.G., Davis, G.B., Davis, F.D.(2003).User Acceptance of information technology:Toward a Unified View. MIS Quarterly, 27(3), 425-478. https://doi.org/10.2307/30036540

Venkatesh, V. (2008). Technology acceptance model 3 and a research agenda on interventions. Decision Sciences, 39(2), 273-315. https://doi. org/10.1111/j.1540-5915.2008.00192.x

Villalobos-Dintrans, P. (2017). Aging and long-term care in Chile: challenges in the OECD context. Revista Panamericana de Salud Publica, 41(9), e86. https://europepmc.org/backend/ptpmcrender. $\underline{\text { fcgi?accid=PMC6660891\&blobtype }=\text { pdf }}$

Welford, C., Murphy, K., Wallace, M. C. D. (2010). A concept analysis of autonomy for older people in residential care. Journal of Clinical Nursing, 19(9-10), 1226-1235. https://doi. org/10.1111/j.1365-2702.2009.03185.x 